\title{
Interpreting and validating the thermal cone penetration test (T-CPT)
}

\author{
PHILIP J. VARDON*, DIMITRIS BALTOUKAS $\uparrow$ and JOEK PEUCHEN $\dagger$
}

\begin{abstract}
The thermal cone penetration test (T-CPT) allows in situ measurement of the thermal properties of soil during a CPT - that is without withdrawing the CPT tool and introducing another tool such as a thermal needle probe. An interpretation method is presented based on one-dimensional axisymmetric analytical solutions. Numerical verification of the method is given and numerical simulations are presented to derive a calibration factor required for the interpretation model. This factor accounts for the temperature sensor location and two-dimensional geometric effects, particularly internal voids and end effects for the cone penetrometer. Finally field data are presented for the T-CPT and compared with results from in situ needle probe tests and laboratory tests on sampled material. The interpretation model is able to provide thermal conductivity values robustly.
\end{abstract}

KEYWORDS: in situ testing; penetrometers; temperature effects

\section{INTRODUCTION}

Heat flow through soil, and resulting temperatures, are important for ground source heat systems (also called shallow geothermal), for high-power electricity cables, where heat dissipation is advantageous, and for pipelines which carry high-viscosity fluids, which increase in viscosity as the temperature reduces. The design of these systems requires accurate input of soil thermal properties.

The thermal cone penetration test (T-CPT) (Akrouch et al., 2016) is a recent in situ test method to determine the thermal properties of soil, competing with the thermal needle probe (ASTM, 2014). Industry has a long history of CPT penetrometers including temperature sensors (see e.g. de Gijt \& van Roekel, 1987), namely temperature cone penetrometers, utilised mainly to measure in situ temperatures. These cones, like all cones, typically heat up during a CPT (ISO, 2012), due to friction between the cone and the soil, and the heat must be dissipated to measure the in situ temperatures accurately. To do this the CPT is interrupted - that is stopped at a selected depth; the temperature in the cone decays as the heat dissipates through the soil. This offers the opportunity to derive the thermal properties of the soil. This is similar to pore pressure dissipation tests (ISO, 2012) for deriving the hydraulic conductivity and consolidation coefficient of soil. Fig. 1 presents an example T-CPT profile and data from a thermal dissipation test (at $11.5 \mathrm{~m}$ depth) are presented in Fig. 2. The temperature drops attributable to a test can be observed coinciding with the solid horizontal lines in Fig. 1. The low values are close to the in situ soil temperature.

The existing interpretation model for deriving thermal conductivity (Akrouch et al., 2016) is based on an empirical approach and equation fitting, utilising the observation that the pore pressure dissipation equation is similar to the thermal dissipation equation. In this paper, a more theoretical

Manuscript received 21 August 2017; revised manuscript accepted 30 July 2018. Published online ahead of print 3 September 2018.

Discussion on this paper closes on 1 December 2019, for further details see p. ii.

Published with permission by the ICE under the CC-BY 4.0 license. (http://creativecommons.org/licenses/by/4.0/)

* Geo-Engineering Section, Delft University of Technology, the Netherlands (Orcid:0000-0001-5614-6592).

$\dagger$ Fugro, the Netherlands. approach to developing an interpretation model is taken and validated against experimental data. An example calculation sheet of the validated interpretation model is available (Vardon et al., 2018).

TEST METHODS FOR SOIL THERMAL PROPERTIES

The T-CPT cone includes a temperature sensor (see Fig. 3). The T-CPT involves pushing the T-CPT cone to a selected depth below the ground surface, during which CPT data (e.g. cone resistance, sleeve friction and pore pressure) are acquired, stopping the cone and measuring the temperature decay inside the cone. No heat source is required. The test is carried out until the interpretation model can yield a good result, or until no further temperature decay is measured, or for any other operational reason.

Akrouch et al. (2016) proposed an empirical interpretation model for the T-CPT, based on the similarities of the thermal conductivity (energy balance) equation to the advective water flow (mass balance) equation. They suggested calibration parameters for these equations based on numerical modelling with a simplified domain and further calibrated against laboratory data. A data reduction to determine a theoretical maximum temperature using a hyperbolic curve to fit the data was undertaken, to determine a characteristic time, which was then used to estimate the thermal properties. The method was seen to be able to reasonably determine the thermal conductivity within a limited number of tests.

The thermal needle probe (ASTM, 2014) is used both in the laboratory and in situ to derive the thermal conductivity of soil (see Fig. 3 for an example schematic representation of an in situ version). The probe works on the principle that heat is dissipated radially and with the application of a certain known power (constant heat flux), the increase in temperature can be analysed to derive thermal conductivity. Both heating and cooling phases can be used. A line source solution (Carslaw \& Jaeger, 1959) is utilised as the basis of the interpretation models (ASTM, 2014). The thermal needle probe is, as the name suggests, thin to reduce disturbing the soil and to satisfy, as far as possible, the assumption of an infinitely thin line source used in the analytical model. This yields a major disadvantage for in situ testing, in that the probe is fragile and can easily be damaged. This limits its practical use in soil with significant strength and at significant depth. Moreover, in situ testing requires the needle probe to be separately pushed into the soil when testing 

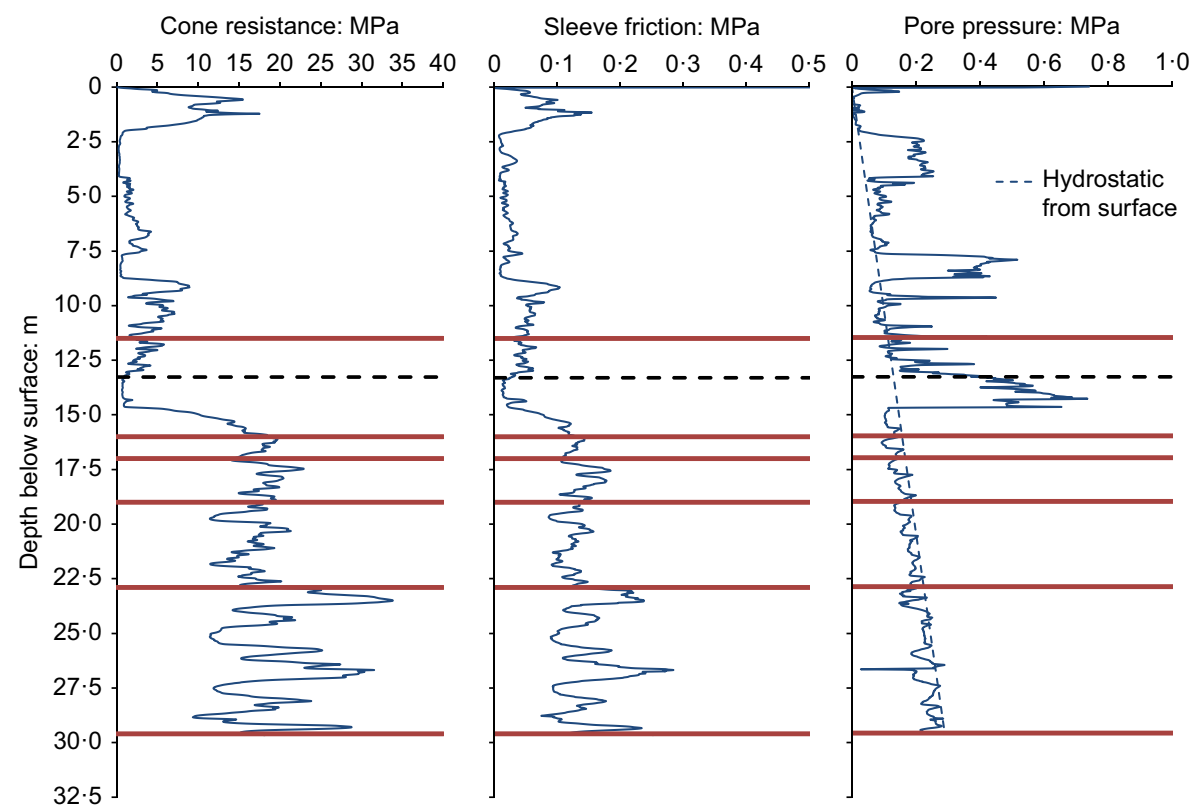

Temperature: ${ }^{\circ} \mathrm{C}$

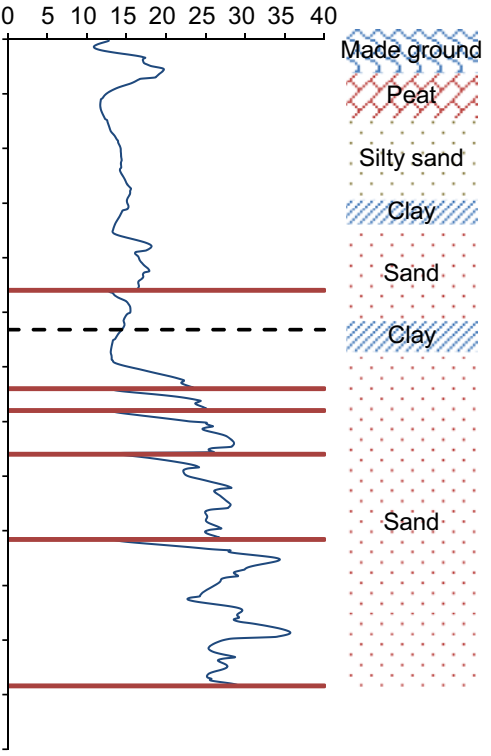

Fig. 1. CPT profile including temperature measured in the cone penetrometer. Solid horizontal lines indicate T-CPT locations and dotted horizontal line indicates a T-CPT at an adjacent location
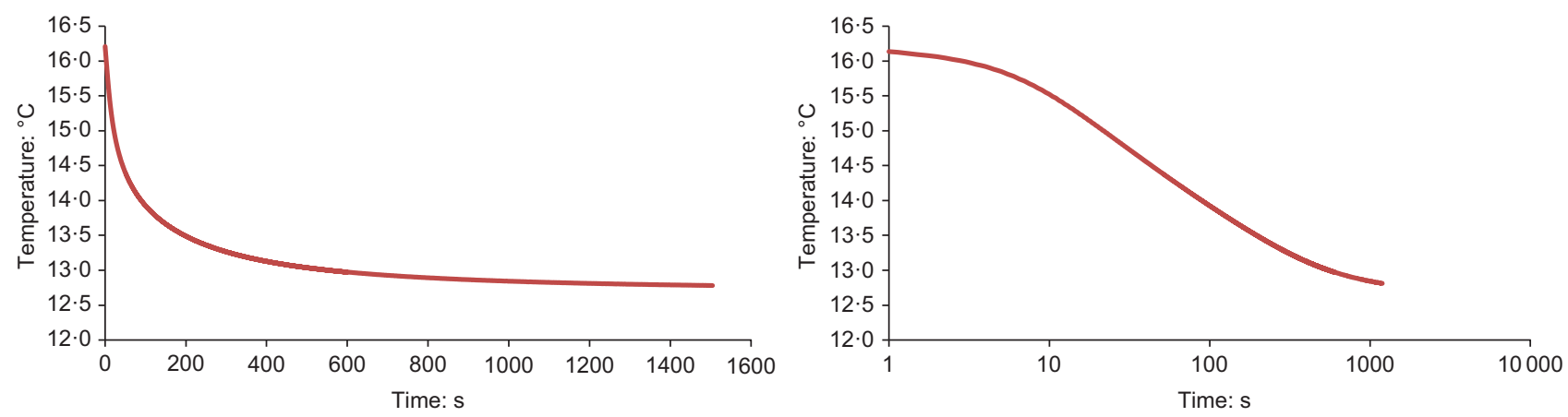

Fig. 2. T-CPT thermal dissipation data at $11.5 \mathrm{~m}$ depth at the location from Fig. 1, on both linear (left) and logarithmic (right) scales

is required, which takes considerable time, extending operations. Alternative probes, based on the same principle of a heater embedded in a cylinder, have been proposed (e.g. Ewen $\&$ Thomas, 1992). The infinite line theory is modified for an appropriate interpretation model; however, operation requires a stable pre-drilled hole, therefore limiting operations.

\section{PROPOSED INTERPRETATION MODEL}

An interpretation model has been developed for (bulk) thermal parameter values of heat conductivity, $k$, and the volumetric heat capacity, $C$. The model is based on axisymmetric conditions and the assumption that the dominant heat transfer process is conduction. As a fixed amount of energy is contained within the T-CPT cone, three previously developed one-dimensional (1D) axisymmetrical analytical solutions (Jaeger, 1956; Carslaw \& Jaeger, 1959) have been used

(a) an instantaneous heat release along a line inside an infinite medium

(b) an instantaneous heat release along the surface of a cylinder inside an infinite medium

(c) a perfectly conducting cylinder inside an infinite medium with an initial temperature different to that of the surrounding medium.

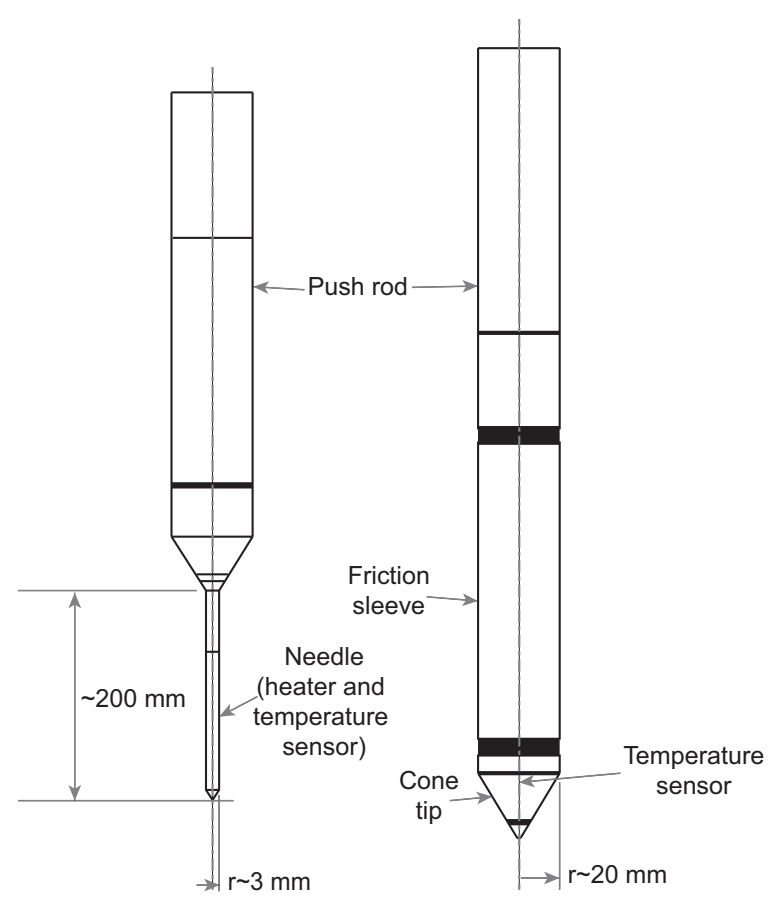

Fig. 3. Thermal needle probe (left) and T-CPT cone penetrometer (right) 
In all three cases this leads to the same solution for thermal conductivity, considering a small radius (e.g. that of a CPT cone) and a long timescale (e.g. $>100 \mathrm{~s}$ for typical soils)

$$
k=\underbrace{f_{\mathrm{TC}} \frac{S\left(T_{\max }-T_{0}\right)}{4 \pi\left[t\left(T-T_{0}\right)\right]}}_{\text {I. Directly from data }}=\underbrace{f_{\mathrm{TC}} \frac{S\left(T_{\max }-T_{0}\right)}{4 \pi \exp \left(i_{T}\right)}}_{\text {II. Graphical method }}
$$

where $f_{\mathrm{TC}}$ is a factor included for calibration of the T-CPT cone; $S=c_{\mathrm{p} \text {,steel }} \rho_{\text {steel }} A_{\text {steel }}$ is the heat content/release per length per degree; $c_{\mathrm{p}}$ is the specific heat capacity; $\rho$ is the density; $A$ is the cross-sectional area of the T-CPT cone; subscript 'steel' attributes properties relevant to steel; $T_{\max }$ is the maximum recorded temperature; $T_{0}$ is the initial ground temperature; $T$ is the temperature at the current time; and $t$ is time. The parameter $i_{T}$ is the $y$-intercept $\left(i_{T}\right)$ from a tangent drawn in the linear portion of a $\ln \left(T-T_{0}\right)-\ln (t)$ plot - that is when time is large. Factor $f_{\mathrm{TC}}$ is included to allow for voids within the cone and the location of the sensor within the cone. A derivation of the proposed solution from the three analytical solutions is presented in the Appendix.

The solution will converge when the time is sufficient and at that time a $\ln \left(T-T_{0}\right)-\ln (t)$ plot will have a gradient of exactly -1 , providing an additional check of the method. The two parts of equation (1) are theoretically equal, but provide the opportunity to either calculate the thermal conductivity directly from the data or by plotting the data in a $\ln \left(T-T_{0}\right)-\ln (t)$ plot.

Equation (1) requires the accurate determination of $T_{0}$, which can be undertaken by waiting until the majority of the heat has dissipated or by way of the following equation

$$
T_{0}=\frac{t_{1} T_{1}-t_{2} T_{2}}{t_{1}-t_{2}}
$$

where the subscript 1 relates to an earlier time and 2 a later time.

Equation (2) uses the same assumptions as equation (1); see the Appendix for details of the derivation.

Only the third of the analytical solutions represents the temperature well in the short term where the heat capacity has the major contribution. This yields a solution for the volumetric heat capacity as

$$
\begin{aligned}
C=\rho c_{\mathrm{p}} & =\underbrace{f_{\mathrm{HC}}\left(\left.\frac{\mathrm{d} T_{\text {norm }}}{\mathrm{d} \sqrt{t}}\right|_{t \rightarrow 0} \frac{S}{4 r_{\mathrm{CPT}}}\right)^{2} \frac{1}{\pi k}}_{\text {I. Directly from data }} \\
& =\underbrace{f_{\mathrm{HC}}\left(\operatorname{grad}_{T} \frac{S}{4 r_{\mathrm{CPT}}}\right)^{2} \frac{1}{\pi k}}_{\text {II. Graphical method }}
\end{aligned}
$$

where $f_{\mathrm{HC}}$ is a factor included for calibration of the T-CPT cone; $r_{\mathrm{CPT}}$ is the radius of the T-CPT cone; $\operatorname{grad}_{T}$ is the gradient at the origin of a $T_{\text {norm }}-\sqrt{t}$ plot and

$$
T_{\text {norm }}=\frac{T_{\max }-T}{T_{\max }-T_{0}}
$$

As for the thermal conductivity, the two parts of equation (3) allow the volumetric heat capacity to be calculated directly from the data or by way of a graphical method. A full derivation of the solution is presented in the Appendix.

It is noted that thermal conductivity and heat capacity can be calculated during a test, as $T_{\max }$ can be determined at the start of the test and $T_{0}$ can be progressively more accurately determined during the test. This implies no substantial post-test data treatment as required in Akrouch et al. (2016).

\section{INTERPRETATION MODEL VERIFICATION \\ $1 D$ verification approach}

The interpretation model presented in the previous section entitled 'Proposed interpretation model' includes a number of simplifications. 1D axisymmetric numerical analysis is presented in the current section to verify the method, using the same assumptions, which means that the calibration factors in equations (1) and (3) are equal to one. The later section entitled 'Determination of calibration factor $f_{\mathrm{TC}}$ ' presents two-dimensional (2D) axisymmetric numerical analysis that is used to calculate the calibration factor $f_{\mathrm{TC}}$. No field data are available for heat capacity measurements and therefore no results of a heat capacity calibration factor $f_{\mathrm{HC}}$ are presented. Both $1 \mathrm{D}$ and $2 \mathrm{D}$ modelling investigations were undertaken using Comsol v5.2. This section also serves as an example of how to use the interpretation method.

\section{$1 D$ numerical model}

The 1D axisymmetric model had a $0.01 \mathrm{~m}$ radius solid steel body (representing a T-CPT cone penetrometer) within a $5 \mathrm{~m}$ radius soil body, which had a thermal conductivity of $2.5 \mathrm{~W} / \mathrm{mK}$ (Table 1 ). Fixed temperature boundary conditions equal to the initial soil temperature were applied at the soil radius.

Two simulations were undertaken, one with an initial cone temperature of $40^{\circ} \mathrm{C}\left(\mathrm{d} T=20^{\circ} \mathrm{C}\right)$ and another with $25^{\circ} \mathrm{C}\left(\mathrm{d} T=5^{\circ} \mathrm{C}\right)$. The temperature of the cone against time for both simulations is shown by the solid lines in Fig. 4 .

\section{Verification of the interpretation method}

In situ temperature. The back-calculated initial in situ temperatures, $T_{0}$ of equation (2), are presented in Fig. 4. It can be seen that, for the $\mathrm{d} T=20^{\circ} \mathrm{C}$ analysis, after approximately $500 \mathrm{~s}$ the value converges to a stable value, accurate to $\sim 0.05^{\circ} \mathrm{C}$, whereas the actual temperature recorded in the cone is still $\sim 1 \cdot 5^{\circ} \mathrm{C}$ above $T_{0}$. For later calculations $T_{0}$ is taken as $20.02^{\circ} \mathrm{C}$, the value at $500 \mathrm{~s}$. $T_{\max }$ is taken as the maximum recorded temperature $\left(40.02^{\circ} \mathrm{C}\right.$ due to a minor numerical oscillation at the start of the analysis). For the lower initial temperature difference analysis $\left(\mathrm{d} T=5^{\circ} \mathrm{C}\right)$, the value of $T_{0}$ converges marginally faster, with accurate values (error $<0.05^{\circ} \mathrm{C}$ ) predicted after $\sim 300 \mathrm{~s}$. The difference in the convergence times is due to both the temperature gradients in the soil and the impact of the heat capacity in an axisymmetric system. It can be seen that the time taken for convergence is not linearly proportional to the temperature difference, agreeing theoretically with the derivations given in the Appendix. For example, the last term in equation (7), which becomes small at large times, is not dependent on the temperature difference. If the allowable error is expressed as a percentage of the initial temperature difference, the

Table 1. Model properties for the numerical simulations

\begin{tabular}{l|l}
\hline Property & Value \\
\hline T-CPT cone radius 1D model, $r_{\mathrm{CPT}}$ & $0 \cdot 010 \mathrm{~m}$ \\
T-CPT cone radius 2D model, $r_{\mathrm{CPT}}$ & $0 \cdot 018 \mathrm{~m}$ \\
Initial soil temperature, $T_{0}$ & $20^{\circ} \mathrm{C}$ \\
Initial T-CPT cone temperature, $T_{\max }$ & $40^{\circ} \mathrm{C}, 25^{\circ} \mathrm{C}$ \\
Soil specific heat capacity, $c_{\mathrm{p}, \text { soil }}$ & $800 \mathrm{~J} / \mathrm{kgK}$ \\
Soil density, $\rho_{\text {soil }}$ & $2000 \mathrm{~kg} / \mathrm{m}^{3}$ \\
Soil thermal conductivity, $k_{\text {soil }}$ & $2 \cdot 5 \mathrm{~W} / \mathrm{mK}$ \\
T-CPT cone specific heat capacity, $c_{\mathrm{p}, \text { steel }}$ & $475 \mathrm{~J} / \mathrm{kgK}$ \\
T-CPT cone density, $\rho_{\text {steel }}$ & $7850 \mathrm{~kg} / \mathrm{m}^{3}$ \\
T-CPT cone thermal conductivity, $k_{\text {steel }}$ & $44.5 \mathrm{~W} / \mathrm{mK}$ \\
\hline
\end{tabular}




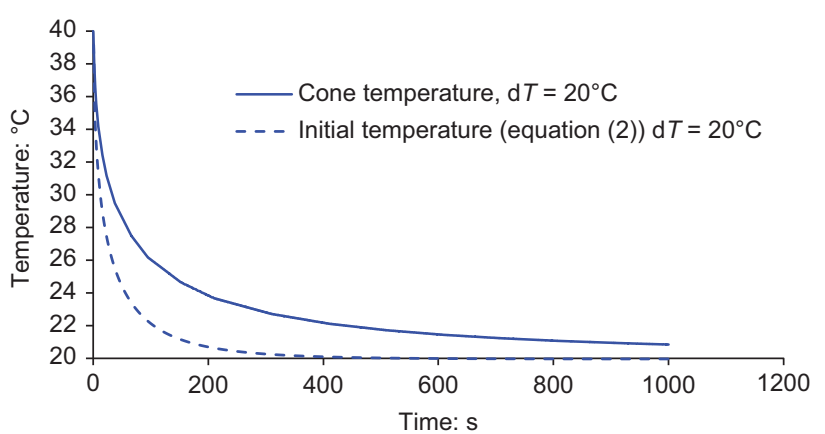

(a)

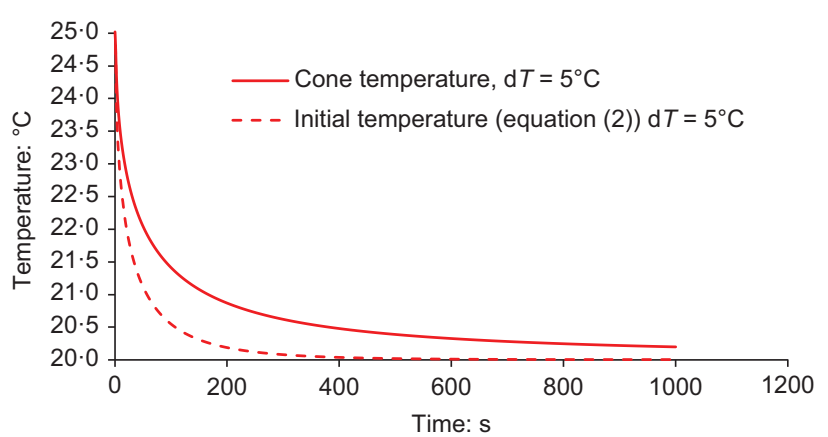

(b)

Fig. 4. Cone temperature and back-calculated initial temperature (equation (2)) from 1D numerical modelling results: (a) with an initial temperature difference of $20^{\circ} \mathrm{C}$; (b) with an initial temperature difference of $5^{\circ} \mathrm{C}$

convergence rate is the same, as can be observed in Fig. 4, where the dotted lines follow exactly the same path (and the solid lines do not), where the axes are fixed at the maximum and minimum temperatures. As $T_{0}$ is initially unknown during tests, a proportional convergence rate, defined as $100\left(T_{0}{ }^{n-1}-T_{0}{ }^{n}\right) /\left(T_{0}{ }^{n} \mathrm{~d} t\right)$, where $n$ is the current reading, is practical as an indicator of convergence. Note that this rate will be affected by thermal conductivity. At $500 \mathrm{~s}$ for the $\mathrm{d} T=20^{\circ} \mathrm{C}$ analysis, this is $0.002 \% / \mathrm{s}$ and at $300 \mathrm{~s}$ for the $\mathrm{d} T=5^{\circ} \mathrm{C}$ analysis, this is $0.003 \% / \mathrm{s}$.

Thermal conductivity. The two methods of calculating the thermal conductivity are presented in Figs 5 and 6. Fig. 5 presents the evolution of the first part of equation (1) in time, where the thermal conductivity is directly calculated from the data. The cone calibration factor is unity here - that is not calibrated. It is seen that the calculated value of thermal conductivity quickly reduces and becomes relatively stable after around $500 \mathrm{~s}$ for both analyses. Only slight differences exist between the two models due to the minor differences in estimation of the initial temperature.

By taking the average between 500 and $600 \mathrm{~s}$, the calculated thermal conductivity is $2.44 \mathrm{~W} / \mathrm{mK}$, which is a $\sim 2.5 \%$ error.

Figure 6 presents the proposed graphical method for the $\mathrm{d} T=20^{\circ} \mathrm{C}$ analysis. The data are plotted on a $\ln \left(T-T_{0}\right)-\ln (t)$ plot, with a tangent to the later linear part drawn to the $y$-intercept. The theoretical gradient of -1 is shown in grey. From the $y$-intercept, using the second part of equation (1), the thermal conductivity can be backcalculated as $k=2.47 \mathrm{~W} / \mathrm{mK}$, an error of $\sim 1 \cdot 2 \%$. An error of $0 \cdot 1$ in the value of the intercept yields an approximately $10 \%$ error in the thermal conductivity value. It is noted that the method is sensitive to $T_{0}$, and the results from an inaccurate $T_{0}$ of $20.2^{\circ} \mathrm{C}$ yield $k=2.77 \mathrm{~W} / \mathrm{mK}$, an error of $\sim 11 \%$. However, when using the incorrect initial temperature, the gradient in the latter part of the test, which should be theoretically -1 , is not linear. This can therefore provide an additional check for the accuracy of the initial temperature and the data used.

Heat capacity. The two methods of calculating the heat capacity are presented in Figs 7 and 8 , with the theoretical value being $c_{\text {p,soil }} \times \rho_{\text {soil }}=1600000 \mathrm{~J} / \mathrm{m}^{3} \mathrm{~K}$. Equation (3), part I was used to estimate the volumetric heat capacity for both analyses, using again a calibration factor of unity, with the results shown in Fig. 7. The forward finite-difference method was used to evaluate the differential, with the time window in the differential calculation being $10 \mathrm{~s}$. It is noted

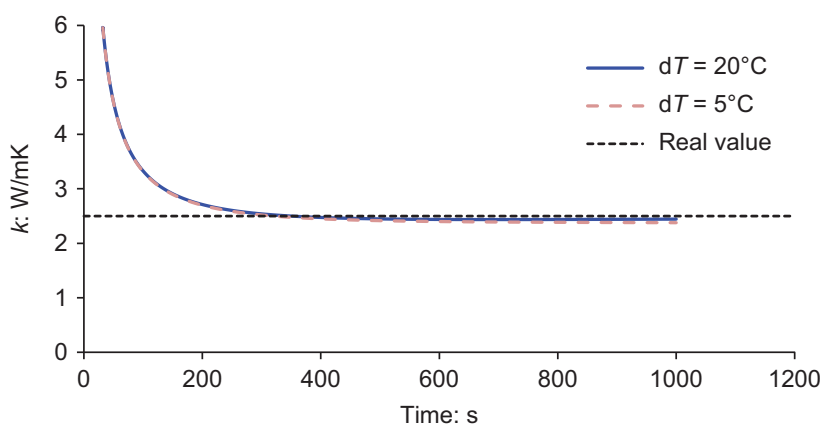

Fig. 5. Back-calculated thermal conductivity $k$ (equation (1) - part I) from 1D numerical modelling results

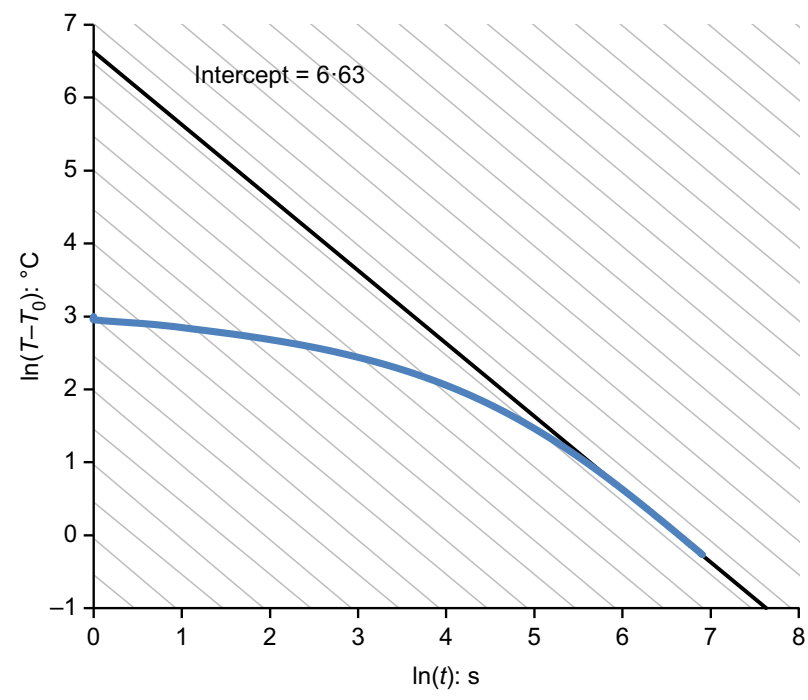

Fig. 6. The $\ln \left(T-T_{0}\right)-\ln (t)$ plot of results with the intercept required for determination of thermal conductivity (equation (1) - part II)

that the initial behaviour, which is of interest, is sensitive to the choice of time window over which the differential is calculated; decreasing the time window over which the differential is calculated first decreases and then increases the intercept, that is the heat capacity at $t=0$, and increases the peak. To match the proposed interpretation model, the volumetric heat capacity should theoretically be the $y$-intercept; however, it does not due to initial redistribution of the heat and the deviation from the assumption of the T-CPT cone having perfect conduction. Averaging the $y$-intercept and the peak, which makes the method less 


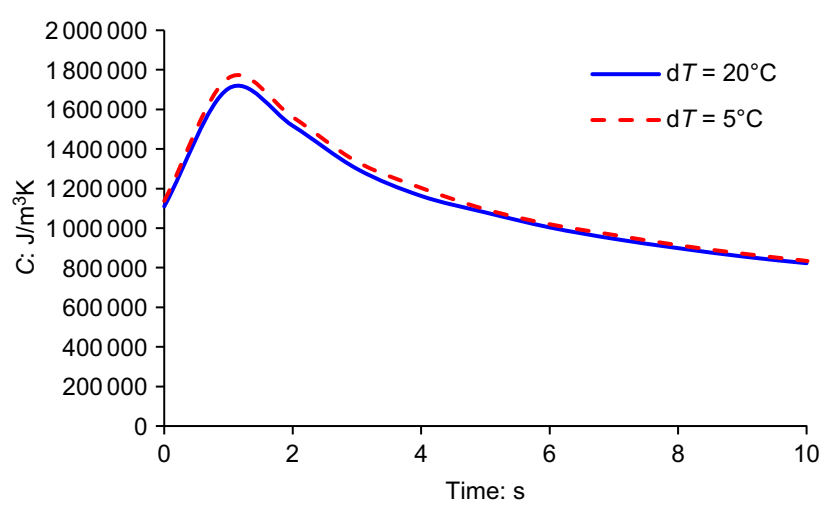

Fig. 7. Back-calculated volumetric heat capacity $C$ (equation (3) part I) from 1D numerical modelling results (time window $=10 \mathrm{~s}$ used to calculate the differential)

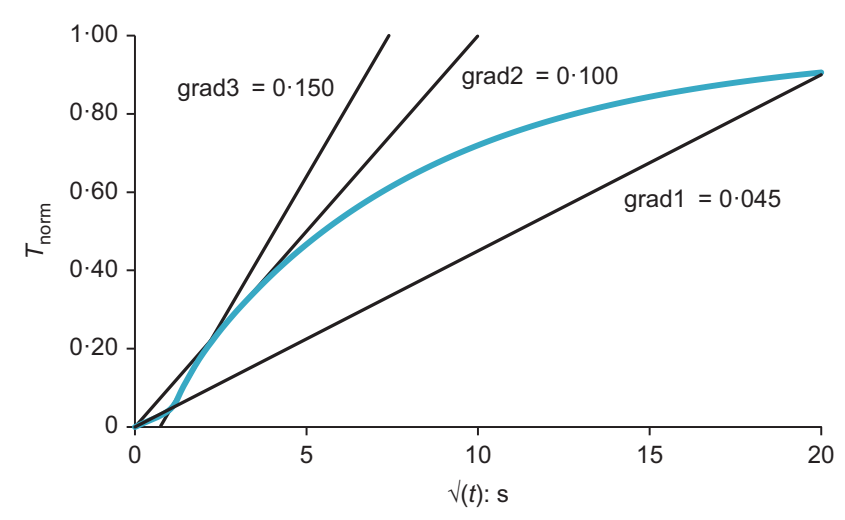

Fig. 8. $T_{\text {norm }}-\sqrt{t}$ plot of results with the gradient required for determination of volumetric heat capacity (equation (3) - part II)

sensitive to the time period used to calculate the differential, yields $\sim 1440000 \mathrm{~J} / \mathrm{m}^{3} \mathrm{~K}$, which is approximately a $10 \%$ error.

The graphical method for the $\mathrm{d} T=20^{\circ} \mathrm{C}$ analysis, which uses a $T_{\text {norm }}-\sqrt{t}$ plot, is presented in Fig. 8 . The maximum gradient does not, as the theory suggests, intercept with the origin. This is due to the initial temperature redistribution and the assumption in the analytical model that the steel is infinitely conductive. Three possible gradients are shown, with each being representative of a solution shown in Fig. 7 . The lowest is the same portion of data which yields the low estimate of $C$ observed at the $y$-intercept, and the highest represents the peak estimate of $C$. It is noted that, owing to the time windows used in calculating the gradients, the values of $C$ calculated are not equal in this case. For example, the lowest gradient (grad1) in Fig. 8 is equal to the intercept in Fig. 7 if a time window of $1 \mathrm{~s}$ is used to calculate the differential; the highest gradient $(\operatorname{grad} 3)$ is equivalent to the peak if the lowest possible time window is used. The graphical figure allows the easy identification of a third gradient (grad2), taking a line from the origin to where it intersects the data, yielding a gradient of $0 \cdot 10$. This is equal to the highest value at the $y$-intercept of Fig. 7, with different time windows used in the differential, in this case about $6 \mathrm{~s}$. The use of the graphical method has two advantages: (a) an easy identification of the different gradients discussed above, and $(b)$ a more straightforward understanding of the square-root time window over which the gradient is taken, as this cannot be constant and pre-defined due to the use of data collected at discrete (and generally evenly spaced) times.
Using the initial gradient (grad1) leads to a large underestimation of $C$ and therefore is not considered further. Using the two other options, the larger gradient $(\operatorname{grad} 3)$ of 0.15 overestimates the energy used and the lower gradient $(\operatorname{grad} 2)$ of $0 \cdot 1$ underestimating it. If the average is used, $0 \cdot 125$, then this gives an estimated volumetric heat capacity of $1728000 \mathrm{~J} / \mathrm{m}^{3} \mathrm{~K}$, which has an $8 \%$ error.

\section{DETERMINATION OF CALIBRATION FACTOR $F_{\mathrm{TC}}$ $2 D$ model}

The 2D axisymmetric model includes a realistic geometry, including interior air-filled voids of the T-CPT cone. The geometry was taken from the T-CPT cone utilised in the later section entitled 'Field data', and has been moderately simplified to reduce the complexity of the mesh - for example, where surfaces are within $0.5 \mathrm{~mm}$ of each other. The geometry of the cone is similar to a majority of CPT cones used commercially; however, there will be specific differences. The air-filled voids have been removed from the mesh due to the very low heat conduction behaviour of air. The mesh has $\sim 5000$ three-noded triangular elements, and is refined around the details of the domain, ensuring that there are at least three elements between any boundaries (material or geometry boundaries). The far boundaries of the domain were selected so as not to influence the results. Fig. 9 presents the details of the domain and mesh.

As in the earlier section entitled '1D numerical model' only heat conduction was modelled as a heat transport mechanism, with the initial temperature in the soil domain being $20^{\circ} \mathrm{C}$. The initial temperature conditions of the T-CPT cone varied, as discussed in the numerical investigation below. The external horizontal boundary has a fixed temperature $\left(20^{\circ} \mathrm{C}\right)$, with all other boundaries having a no-flow condition. Material properties for the steel and soil are as presented in Table 1 and, with the exception of the T-CPT cone details, are the same as in the earlier section 'Interpretation model verification'.

The theoretical calibration factor, $f_{\mathrm{TC}}$, due to the voids is 0.66 (calculated as the ratio of the volume of steel of the $\mathrm{T}-\mathrm{CPT}$ cone to the volume of a cylinder with the same height and radius).

The thermal dissipation has been recorded in the T-CPT cone at three locations, indicated in Fig. 9

(a) cone tip - in the centre of the cone, $20 \mathrm{~mm}$ above the cone apex; this is the location of the temperature sensor of the T-CPT cone used in the later section entitled 'Field data'

(b) mid-height centre $-1 \mathrm{~mm}$ from the inside edge of the steel $(r=7 \mathrm{~mm}), 120 \mathrm{~mm}$ above the cone apex

(c) mid-height edge $-1 \mathrm{~mm}$ from the outside edge of the cone $(r=17 \mathrm{~mm}), 120 \mathrm{~mm}$ above the cone apex.

The modelling investigation comprised two main cases

case A: initial increased cone temperature $\left(\mathrm{d} T=20^{\circ} \mathrm{C}\right)$ case B: heat-source-induced temperature increase followed by dissipation

case B1: heat source applied on the cone tip $\left(5000 \mathrm{~W} / \mathrm{m}^{2}\right)$ followed by dissipation case B2: heat source applied on the friction sleeve $\left(500 \mathrm{~W} / \mathrm{m}^{2}\right)$ followed by dissipation.

Case B provides a better representation of the initial temperature distribution inside the cone at the start of the dissipation test than case A. The heat sources of case B were chosen to achieve a significant temperature rise (similar to 


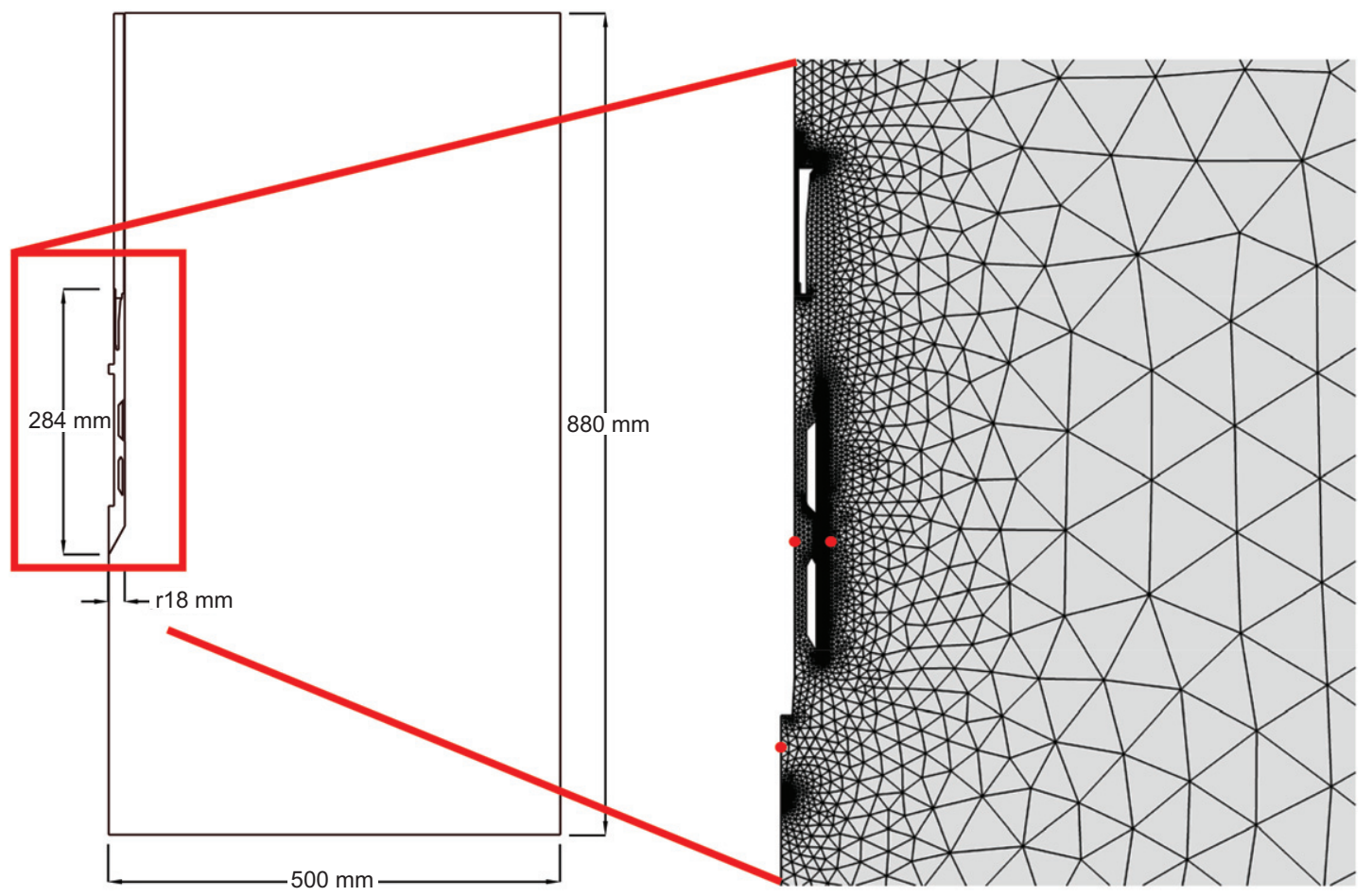

Fig. 9. Domain and mesh details of 2D model

that observed in practice) and ten times higher on the cone tip than on the friction sleeve to represent the significantly higher stresses between the cone tip and the soil than between the friction sleeve and the soil. The soil part of the domain was not active during the part of the analysis where the heat source was applied and was activated during the thermal dissipation part. A sensitivity study has been undertaken on case B1 varying the thermal conductivity of the soil.

\section{Case A: Initial increased T-CPT cone temperature}

The T-CPT cone and push rod were given an elevated temperature of $40^{\circ} \mathrm{C}\left(\mathrm{d} T=20^{\circ} \mathrm{C}\right)$. The early temperature evolution within the T-CPT cone is seen in Fig. 10(a) to be strongly affected by the voids in the cone, with the parts of the cone 'insulated' from the soil by voids showing higher temperatures and lower temperatures in the parts of the cone in between the soil and the voids.

At later times, illustrated in Fig. 10(b) at $500 \mathrm{~s}$, there is little impact of the voids, but there is a reduced temperature in the T-CPT cone tip, showing $2 \mathrm{D}$ effects. The overall heat flow, however, is still seen to be radial, as demonstrated by the almost vertical contours. A calibration factor of less than unity would be expected, due to the voids - that is a lower initial heat content, and if the temperature sensor is located towards the tip.

The calibration factor, $f_{\mathrm{TC}}$, was back-calculated for the three sensor locations, using equation (1) - part I. Results are shown in Table 2. It is seen that the calibration factor ranges from 0.5 to 0.75 depending on where the sensor is located. The cone in the numerical model has a solid volume of $19080 \mathrm{~mm}^{3}$, compared to a cylinder of the same length and radius of $28910 \mathrm{~mm}^{3}$, giving a theoretical averaged area of steel of 0.66 . At the mid-height of the cone in the centre of the cone, the area of steel factor is almost identical to the theoretical value. The differences in $f_{\mathrm{TC}}$ are due to the voids not being located evenly, and due to end effects. At the soil/steel interface the thermal behaviour is influenced both by the steel and the soil conductivity. Interpretation of data from a temperature sensor at the interface would be least reliable.

\section{Case B: Friction-induced temperature increase}

As the T-CPT cone is considered to be used where significant temperatures are generated due to friction, this is considered a more realistic case than the case of initial increased T-CPT cone temperature. The first model case (case B1), where friction is generated on the cone tip, is considered typical for sand (Post \& Nebbeling, 1995). Fig. 11(a) shows the temperatures after $10 \mathrm{~s}$ of the thermal dissipation part of the test. It is seen that the tip area of the T-CPT cone has a temperature elevated above the rest of the T-CPT cone, which is likely to lead to faster dissipation from the cone tip and therefore a lower calibration factor calculated. Fig. 11(b) shows the same analysis after $500 \mathrm{~s}$. The temperature distribution is still uneven, with higher temperatures exhibited near the cone tip.

The calibration factor for a sensor at the tip $(0 \cdot 38$, Table 2$)$ is shown, as expected, to be lower than the theoretical value. At the mid-height in the centre it is closer to the theoretical value and also similar to the modelling where an initial temperature difference is prescribed.

For stiff clays a more likely situation is thought to be heat generation by way of friction on the friction sleeve, case B2. This has been simulated, with again the back-calculated calibration factors shown in Table 2. The results are similar to the case of initial increased T-CPT cone temperature (case A), with the exception that the push rod is significantly cooler.

Sensitivity analysis for thermal conductivity of the soil

The numerical model with heat generation at the cone tip (case B1) has been selected for a sensitivity analysis against thermal conductivity of the soil. This is in part due to the consideration that this heat generation method approaches actual test conditions, in part that it is in these conditions the 


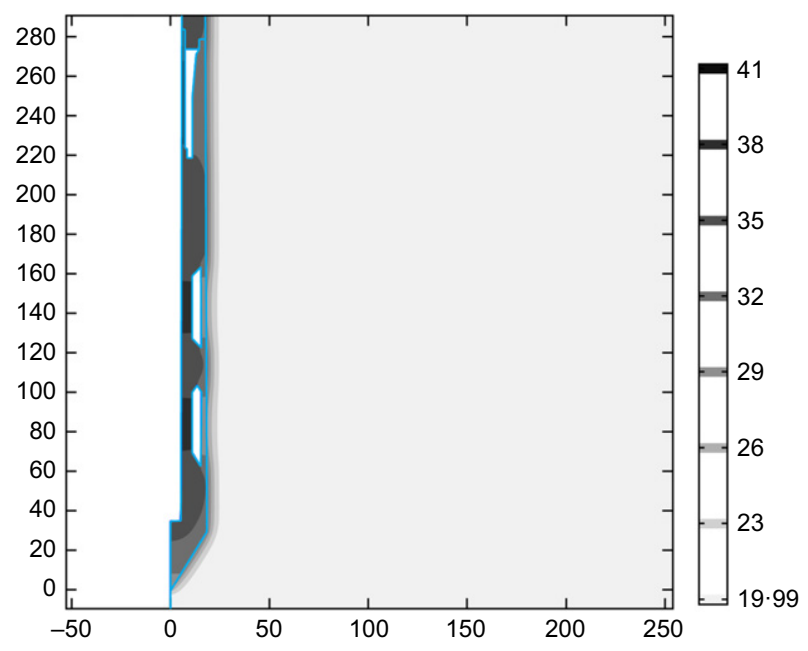

(a)

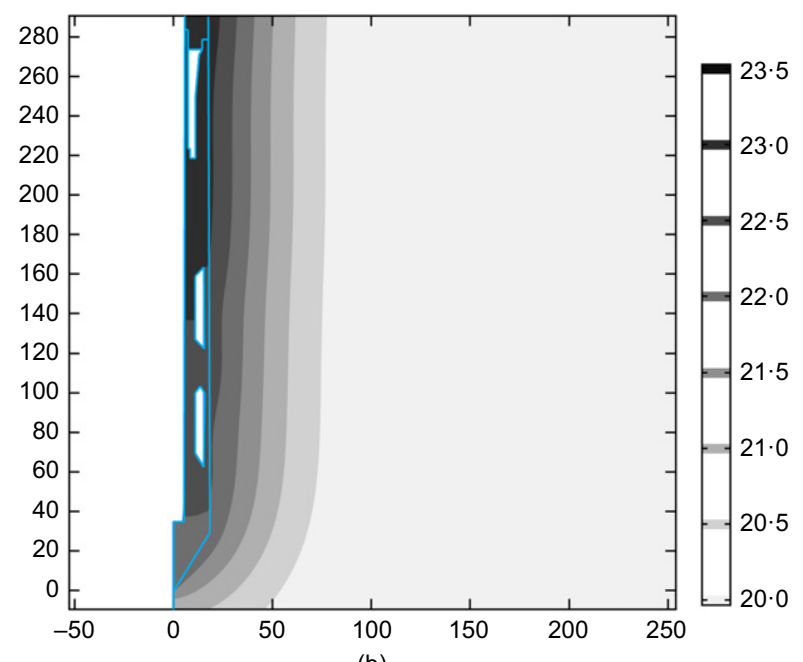

(b)

Fig. 10. Contour plot of temperature $\left(\right.$ in ${ }^{\circ} \mathrm{C}$ ) for the $2 \mathrm{D}$ model case $\mathrm{A}$ with uneven initial temperature conditions: (a) at $10 \mathrm{~s}$; (b) at $500 \mathrm{~s}$. Axes are in $\mathbf{m m}$

Table 2. Back-calculated calibration factor for thermal conductivity

\begin{tabular}{l|l}
\hline Analysis & $f_{\mathrm{TC}}$ \\
\hline Theoretical & 0.66 \\
Initial increased temperature of T-CPT cone (case A) & 0.50 \\
$\quad$ Cone tip & 0.65 \\
$\quad$ Mid-height centre & $0 \cdot 75$ \\
$\quad$ Mid-height edge & 0.38 \\
Initial temperature from friction on cone tip (case B1) & 0.68 \\
$\quad$ Cone tip & $0 \cdot 78$ \\
Mid-height centre & 0.56 \\
Mid-height edge & 0.60 \\
Initial temperature from friction on friction sleeve (case B2) & 0.68 \\
Cone tip & \\
Mid-height centre & \\
Mid-height edge &
\end{tabular}

needle probe cannot be used, and in part that the calibration factor values have the widest range. The thermal conductivity of the soil has been selected within realistic ranges ( 1 to $3 \cdot 5 \mathrm{~W} / \mathrm{mK})$.

The calculated calibration factors $f_{\mathrm{TC}}$ are presented in Fig. 12. It is seen that the factors increase slightly between thermal conductivities of 1 and $2 \mathrm{~W} / \mathrm{mK}$, but are constant

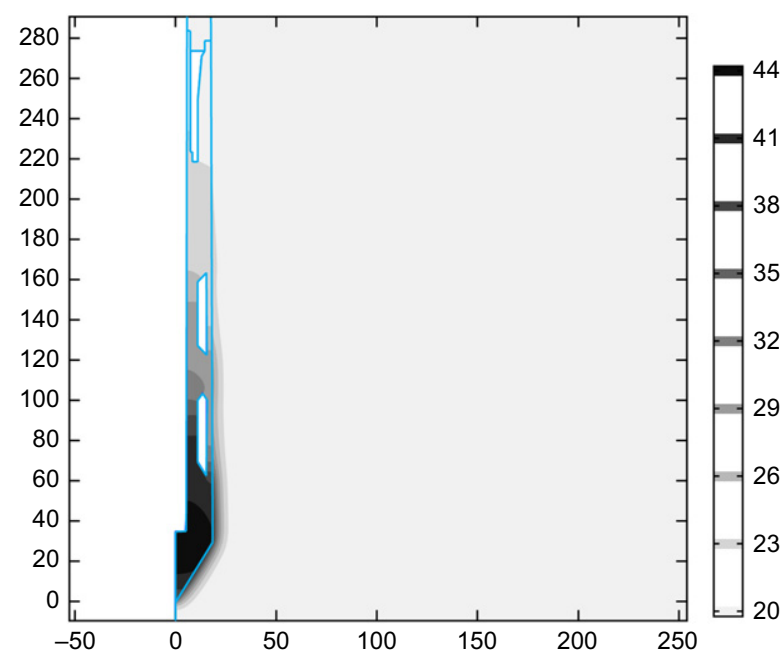

(a)

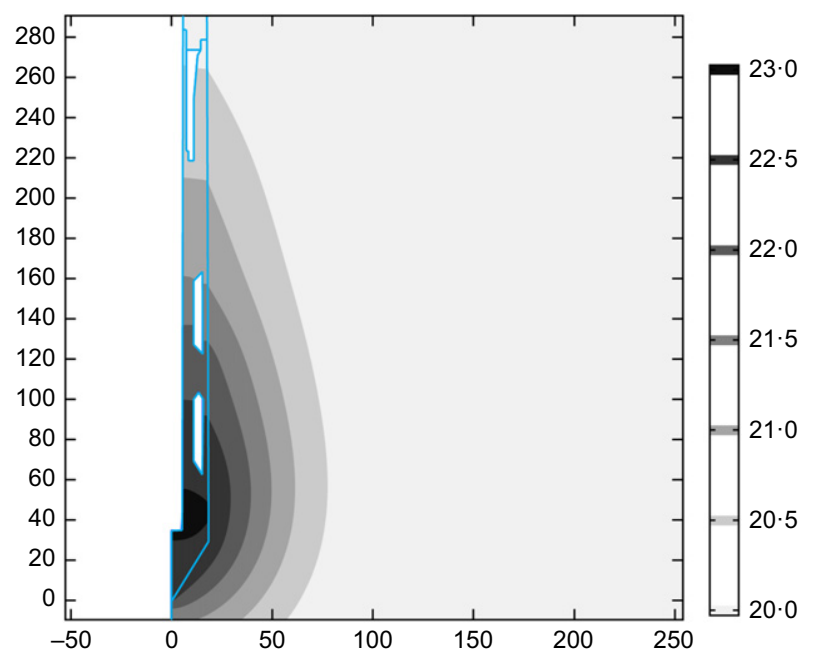

(b)

Fig. 11. Contour plot of temperature (in ${ }^{\circ} \mathrm{C}$ ) for the $2 \mathrm{D}$ model case B1 with temperature generated by way of friction on the cone tip: (a) at $10 \mathrm{~s}$; (b) at $500 \mathrm{~s}$, after the dissipation part of the test has begun. Axes are in $\mathbf{m m}$

thereafter. The ranges of the factors are relatively small. In many cases soil thermal conductivities of $>2 \mathrm{~W} / \mathrm{mK}$ would be expected and a constant value could be assumed. Care should be taken for unsaturated soil conditions where lower values may occur. It is also noted that a temperature sensor at the mid-height centre location is seen here by way of numerical simulation to be favourable, as the calibration factor becomes less sensitive to the heat generation method and close to the theoretical value, although this has not been tested experimentally. This lower sensitivity is due partly to $2 \mathrm{D}$ end effects and partly due to the specific arrangement of the voids in the cone utilised. Generalising the findings, the sensor is proposed to be considered to be placed as far as is practicable away from the voids and at a reasonable distance from the tip. However, moving the sensor further from the heat source may lead to lower differences in measured temperature and therefore lower applicability of the test.

\section{Other considerations}

Change in soil conditions. The T-CPT cone is pushed through the soil, which implicitly means that the soil deforms, and therefore may cause a change in thermal 


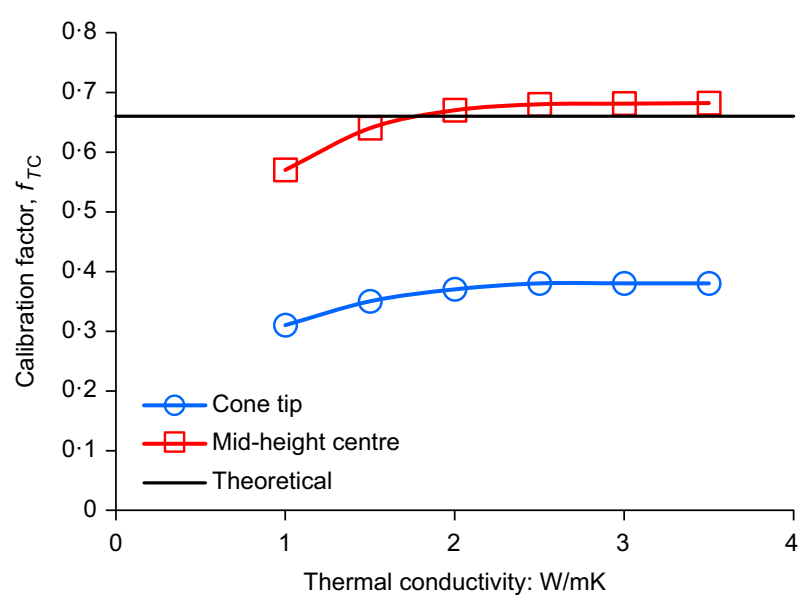

Fig. 12. Calibration factor for sensor locations at the cone tip and at mid-height in the T-CPT cone, case B1

properties (ASTM, 2014). An advantage of using the T-CPT cone to displace the soil is that the thermal connection between the soil and the cone is good. The deformation is fast due to the penetration rate (ISO, 2012), which frequently means that soil suffers only limited volumetric deformation initially. However, during a test, local consolidation may occur and therefore changes in volume leading to changes in thermal properties. This is a complex thermo-hydro-mechanical process and has not been considered in detail in this work. For saturated clayey soils the consolidation process will take place over a longer period than the thermal dissipation test, due to low hydraulic conductivity; therefore, no significant influence from soil volume change is expected. For sandy soils and unsaturated clayey soils, soil volume change may take place during the penetration phase and during the thermal dissipation test. This is a complex issue which deserves some future attention. However, the effect is thought to be limited, mainly as due to the axisymmetric conditions which prevail, significant differences will only occur very close to the cone, and therefore only in a small portion of the soil volume. Moreover, as initially the heat is contained in the cone, not the soil, fast advective transport of water will not contain any of the excess heat, and the impact of advection will reduce as time progresses. This does, however, indicate that the method may not be reliable at very low temperature differences between the cone and the ground, as the heat transfer can be more influenced by the region very close to the cone.

In unsaturated soil conditions the temperature gradient generated in the soil can lead to water vapour movements (e.g. Ewen \& Thomas, 1992). The relatively short timescales (hundreds of seconds) and relatively low temperatures limit this effect.

Non-uniform temperature distribution. As shown in the earlier section 'Case B: Friction-induced temperature increase', how the temperature is generated in the T-CPT cone can affect the calibration factor $f_{\text {TC }}$. The recorded cone resistance and sleeve friction will enable this to be broadly assessed.

Heat is generated as the T-CPT cone moves through the layers above the position of the thermal dissipation test. This means that soils which are stratified, and even soil layers which do not generate enough heat (low friction), can be tested given enough overall heat generation. Soil stratification may result in some differences in the initial temperature distribution in the cone if the layers are significant in thickness. Moreover, if the T-CPT cone during a thermal dissipation test is not within a uniform soil layer, a result will be gained which is affected by heat transport in both layers.

\section{FIELD DATA \\ Test programme}

The extensive test programme covered four different experimental methods. These are: $(a)$ T-CPT; $(b)$ in situ thermal needle probe; $(c)$ thermal needle probe on sampled material, tested immediately after sampling; and $(d)$ thermal needle probe on sampled material in the laboratory, including undisturbed, reconstituted and multiple density tests. The thermal needle probe tests undertaken immediately after sampling were undertaken outside laboratory conditions, but without transportation disturbance - for example, additional change of moisture or changes in density.

The T-CPT method was undertaken including pore pressure measurement (i.e. a piezocone penetration test) according to ISO (2012), stopped at the selected test depth, and the temperature was recorded. The thermal needle probe tests were performed according to ASTM (2014).

The needle probe tests do not result in heat capacity measurements and therefore the T-CPT calculated heat capacity results cannot be verified and will form a future investigation.

The soil profile at the test locations was mainly sand, but at some locations a clay is located close to the ground surface. Over 500 tests were made: $\sim 240$ T-CPTs, $\sim 20$ in situ thermal needle probe tests, $\sim 20$ thermal needle probe tests on samples tested immediately after sampling and $\sim 270$ laboratory thermal needle probe tests. This allowed a comprehensive analysis of the ability of the T-CPT to derive thermal conductivity. However, due to the restrictions of in situ needle probe testing, only a limited number of test results at exactly the same depth were obtained, at the expense of several needle probe instruments during the testing programme.

\section{Results}

Figure 13 presents a selection of results taken at five locations. The calibration factor for the T-CPT $f_{\mathrm{TC}}=0.35$ was used in all cases, using Fig. 12, matching the temperature sensor location (cone tip) and an a priori best estimate of the thermal conductivity of $2 \mathrm{~W} / \mathrm{mK}$. An iterative procedure could be used to correct for this - that is the calibration factor matching the a priori best estimate can be initially used and if the resulting thermal conductivity does not match, the factor can be updated. The five locations have been selected where comparative data are available at the same location. It should be noted that the thermal needle probe/T-CPT tests and the soil samples were generally a few metres apart horizontally. In Fig. 13(d), the sub-locations are up to $10 \mathrm{~m}$ apart. In general, good agreement is seen between the in situ methods, with laboratory tests generally exhibiting a slightly lower measured thermal conductivity than the in situ data consistent with slight de-saturation and a reduction in density of the samples. The multi-density laboratory results show significantly more scatter, as is expected, exhibiting the importance of measuring $k$ at the correct density.

Locations 1 to 4 of Fig. 13 are mainly sandy soil profiles, with clay, clayey or silty material present at the surface at locations 2 to 4 . These locations are fully saturated with the water table above the ground surface. Location 5 is the location with the CPT profile presented in Fig. 1 and the thermal dissipation for the $11.5 \mathrm{~m}$ test given in Fig. 2. The water table is close to, but below, the surface in this location. The test results and interpretation for both the needle 


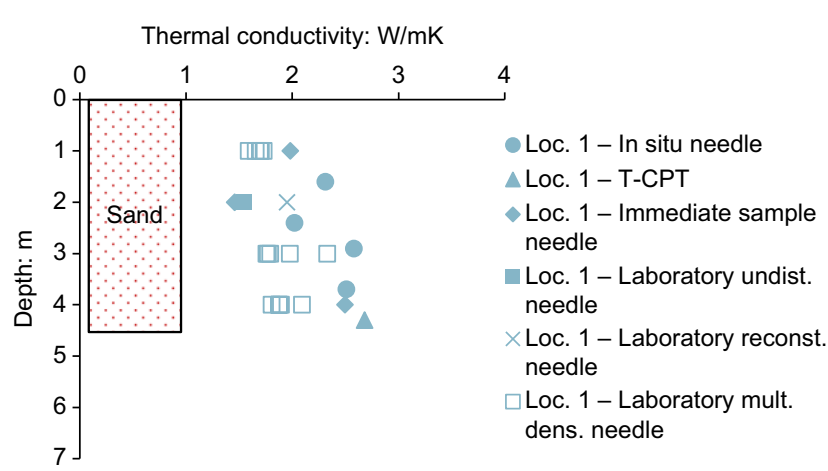

(a)

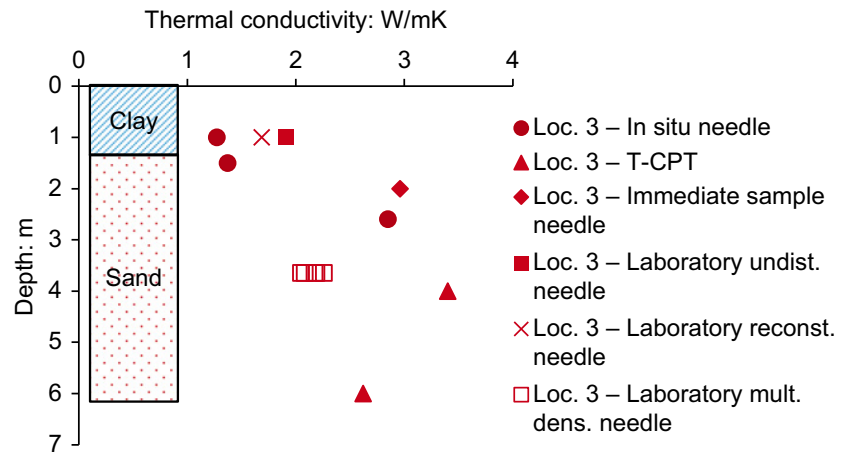

(c)

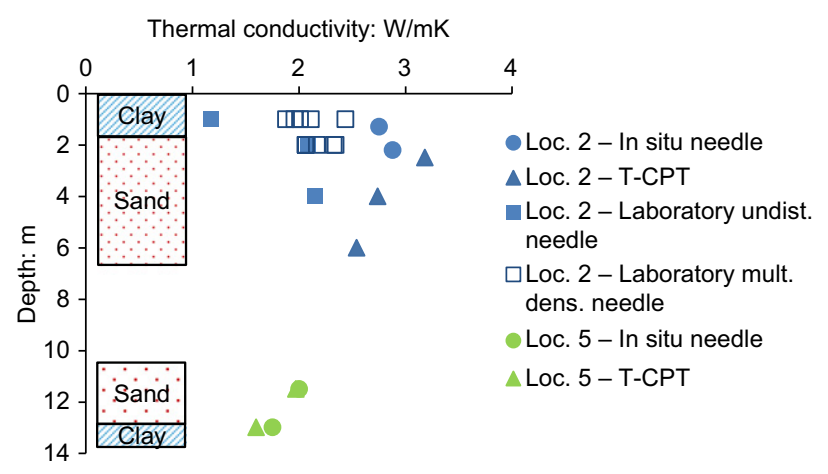

(b)

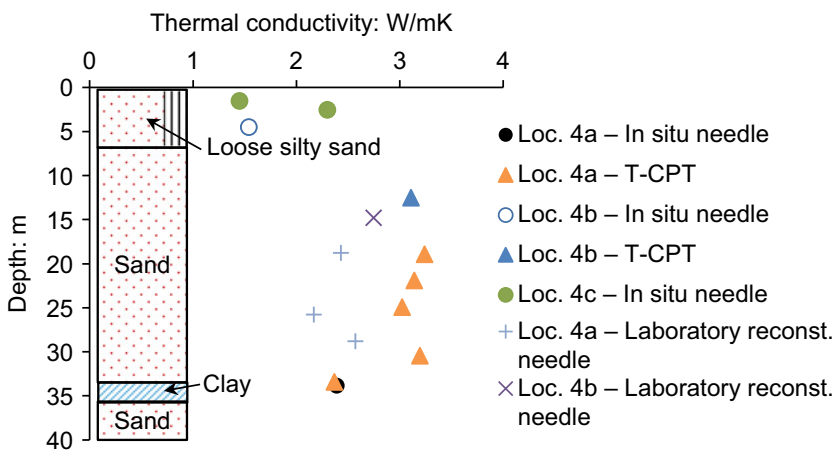

(d)

Fig. 13. (a)-(d) Thermal conductivity results of the different test methods at selected locations. Methods used, in addition to the T-CPT, were an in situ needle probe, and needle probe tests undertaken on sampled material taken in different conditions: immediately tested after sampling (Immediate sample needle), tested in the laboratory with undisturbed samples (Laboratory undist. needle), tested in the laboratory with reconstituted samples (Laboratory reconst. needle) and tested in the laboratory with the sample prepared to multiple densities (Laboratory mult. dens. needle)

probe and T-CPTs at location 5, 11.5 $\mathrm{m}$ depth, are presented in Fig. 14 to demonstrate the method.

Figure 13(a) shows excellent agreement between the in situ tests (thermal needle probe and single T-CPT). The thermal needle probe test taken on site immediately after sampling and the undisturbed laboratory test agree well, and are slightly lower than the in situ data, consistent with slight de-saturation and a reduction in density of the samples. The reconstituted laboratory test, taken at $2 \mathrm{~m}$ depth, had a dry density of $1.5 \mathrm{Mg} / \mathrm{m}^{3}$ and was consistent with the in situ tests. The dry density recorded in the laboratory from the samples taken at this depth was $1 \cdot 1 \mathrm{Mg} / \mathrm{m}^{3}$ compared to dry densities of $>1.5 \mathrm{Mg} / \mathrm{m}^{3}$ calculated using CPT correlations for the in situ tests. The multi-density tests, as expected, cover a range, and the values for the higher density approach the values measured in situ.

Figure 13(b) shows two different locations, one close to the surface, up to $6 \mathrm{~m}$ depth (location 2), and the other between $11 \mathrm{~m}$ and $14 \mathrm{~m}$ (location 5). For both locations excellent agreement between in situ tests was observed. In the upper location, again lower results from laboratory tests are seen, and additionally a reduction of thermal conductivity close to the surface is seen, consistent with a clay soil composition and very low recorded dry density $\left(0.9 \mathrm{Mg} / \mathrm{m}^{3}\right)$.

Figure 13(c) shows again excellent in situ agreement. The laboratory tests present a slightly higher result close to the surface compared to the in situ thermal needle probe results. It is possible that this material was very loose in situ and densified during sampling and transport to the laboratory. Note that the in situ needle probe provides no indication of in situ soil density.

Figure 13(d) shows results over a much greater depth. A $35 \mathrm{~m}$ borehole was drilled, so that a thermal needle probe test could be undertaken below the bottom of the borehole.
Again excellent in situ agreement was found. A clear profile was found with combined needle probe and T-CPT results covering the whole depth. Again, results from samples were shown to be lower, coinciding with laboratory dry densities that were lower than estimated in situ dry densities.

In all cases thermal conductivity profiles are noted - that is the thermal conductivity changes over the soil depth profile. This is consistent with changes in density (generally increasing, due to increasing overburden stresses), and with a variation due to soil material changes. It is also seen that, due to sampling, in most cases, thermal conductivity was measured to be lower than in the in situ tests. This is consistent with sample disturbance and sample de-saturation. It was also seen that if the T-CPT temperature rise was less than $1{ }^{\circ} \mathrm{C}$ an unrealistically high thermal conductivity was calculated (see the near-surface results on Fig. 15(a)). However, such a small temperature rise is often encountered in soft soil conditions where the needle probe can be used. The presented cases show sufficient heat generation by going through sand or sandy layers. This was due to the specific stratigraphy at locations $1-4$, where non-sandy, very soft layers were only encountered at the surface. Therefore, the heat generation mechanism for clays has not yet been validated in this work.

The results for all of the tests are presented in Fig. 15. Fig. 15(a) shows that only a few in situ thermal needle probe tests were undertaken, in particular at significant depth, as these require first borehole drilling, then insertion of the thermal needle probe, which is expensive and difficult to achieve without loss or damage. The T-CPT can easily obtain results at these depths. In Fig. 14(b) there is a weak non-linear correlation between thermal conductivity and recorded soil density, as would be expected for saturated sands. The densities from the T-CPT measurements were estimated using the Mayne et al. (2010) correlation. The weak non-linear 


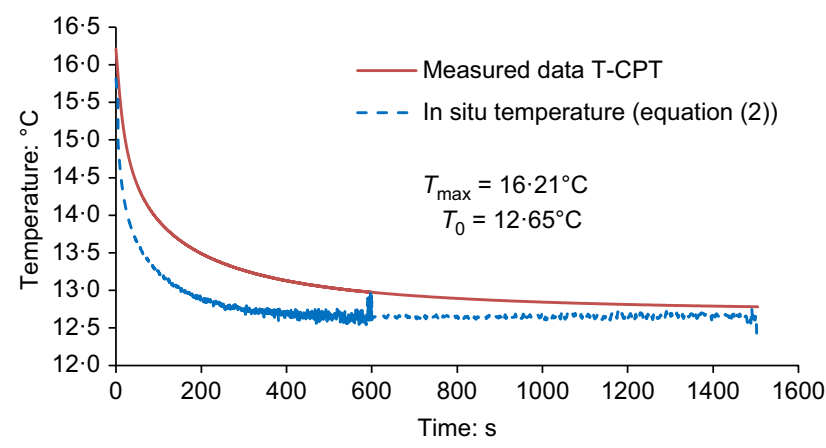

(a)

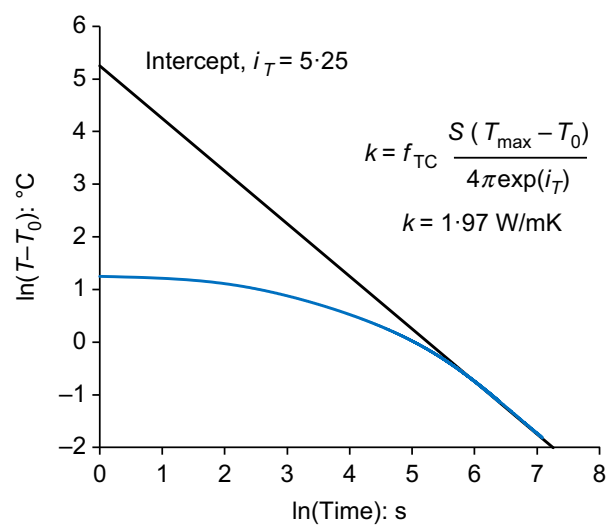

(c)

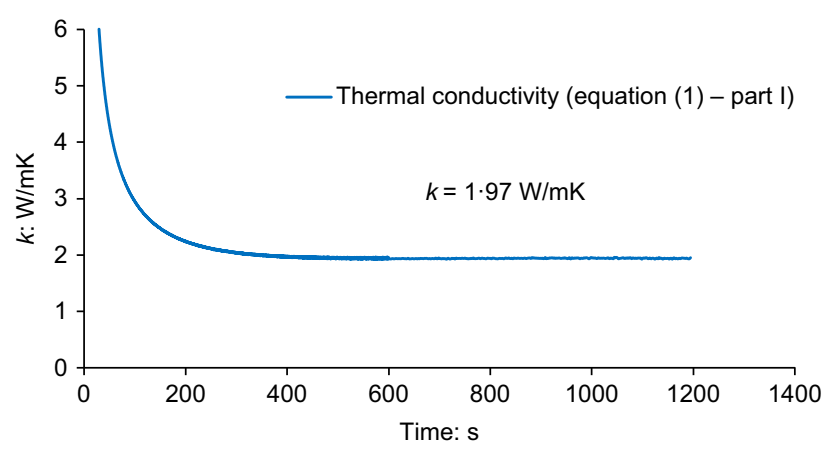

(b)

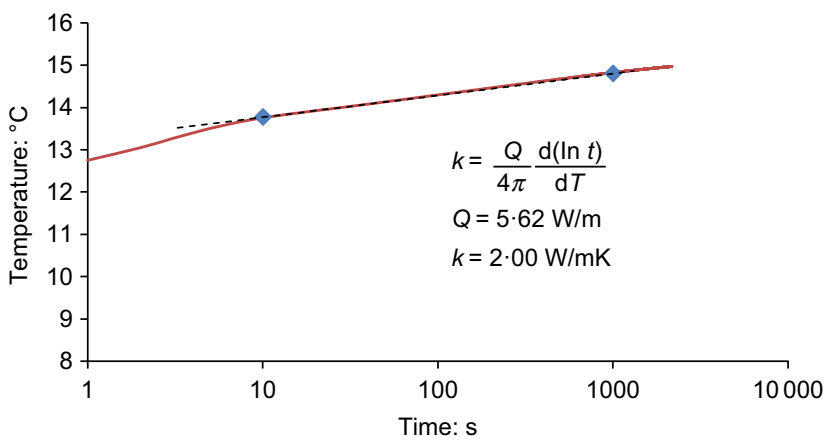

(d)

Fig. 14. Thermal conductivity determination at location 5, 11.5 m depth: (a) measured data and initial in situ temperature determination from T-CPT using equation (2); (b) thermal conductivity determined from T-CPT using equation (1) - part I; (c) thermal conductivity determined from T-CPT using equation (1) - part II; and (d) thermal conductivity determined from the in situ needle probe

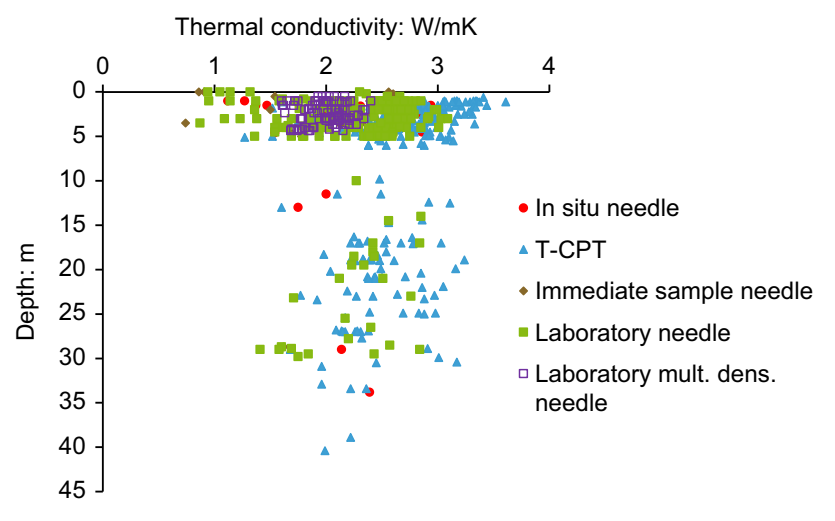

(a)

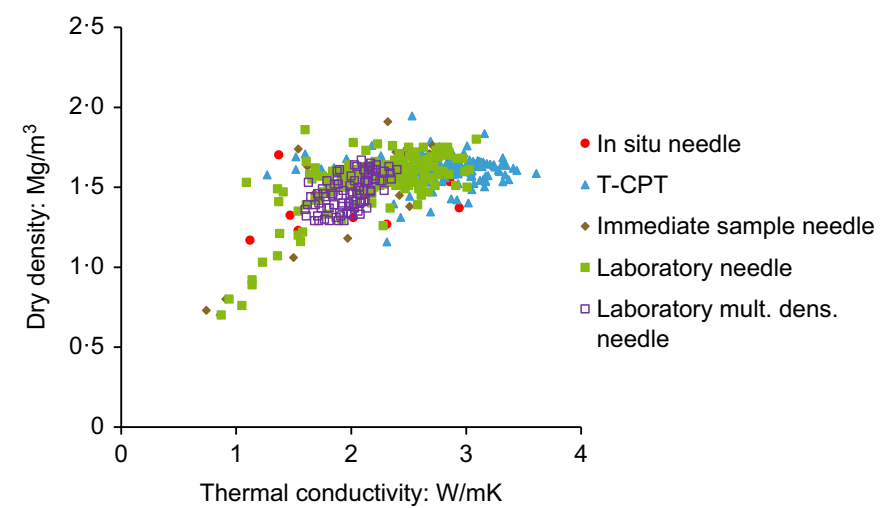

(b)

Fig. 15. Thermal conductivity results of the different test methods: (a) plotted against depth; (b) plotted against dry density

correlation is consistent with, for example, the thermal conductivity calculated with a water and solid geometric mean, as opposed to an arithmetic mean, which would give a linear correlation (Farouki, 1981). Scatter on this plot is thought to be due to composition differences in the soil and test limitations. It is noted that, at dry densities of about $1.5 \mathrm{Mg} / \mathrm{m}^{3}$ and above, a large thermal conductivity range can be expected, with only minor dry density differences. This implies that density correlations would not be able to act as a proxy for thermal conductivity.

A single cone penetrometer has been tested, with a single sensor location, therefore this specific location can be considered fully validated. An excellent agreement with numerical modelling was found, which indicates other locations would also perform well.

\section{CONCLUSIONS}

This paper presents a robust interpretation model for deriving in situ thermal conductivity of soil from a cone penetrometer equipped with a temperature sensor. The test method (T-CPT) relies on a temperature rise in the steel of the penetrometer during a CPT and subsequent measurement of temperature decay during a pause in penetration. The presented interpretation model, which easily suits spreadsheet-type data analysis, relies on a theoretical analytical model and a one-off numerical calibration of the specific cone penetrometer used for data acquisition. The interpretation model was successfully tested against field data, where sufficient heat was generated. Between the T-CPT and the thermal needle probe the majority of ground conditions can be tested. 
The thermal needle probe can test very soft, close-tosurface, soil layers and other soil layers can be tested by the T-CPT.

\section{ACKNOWLEDGEMENT}

The funding of this work and provision of all experimental data by Fugro are gratefully acknowledged.

\section{APPENDIX. DERIVATION OF ANALYTICAL METHODS}

\section{Heat conduction}

To determine a theoretical formulation for the T-CPT based upon heat conduction, it is assumed that there is no convection in the timescales investigated and therefore (bulk) thermal parameter values for the material are derived.

The heat conduction equation, written in 1D axisymmetric coordinates is

$$
\frac{\partial T}{\partial t}=\frac{k}{c_{\mathrm{p}} \rho}\left(\frac{\partial^{2} T}{\partial r^{2}}+\frac{1}{r} \frac{\partial T}{\partial r}\right)
$$

where $T$ is the temperature; $t$ is time; $k$ is the thermal conductivity; $c_{\mathrm{p}}$ is the specific heat capacity; $\rho$ is the density; and $r$ is the radial coordinate.

Unlike a thermal needle probe, where a constant flux boundary condition is used, in this case a fixed amount of energy is contained within the T-CPT cone. There are three similar situations which can be analytically determined (Jaeger, 1956; Carslaw \& Jaeger, 1959). Each is elaborated below

(a) an instantaneous heat release along a line inside an infinite medium

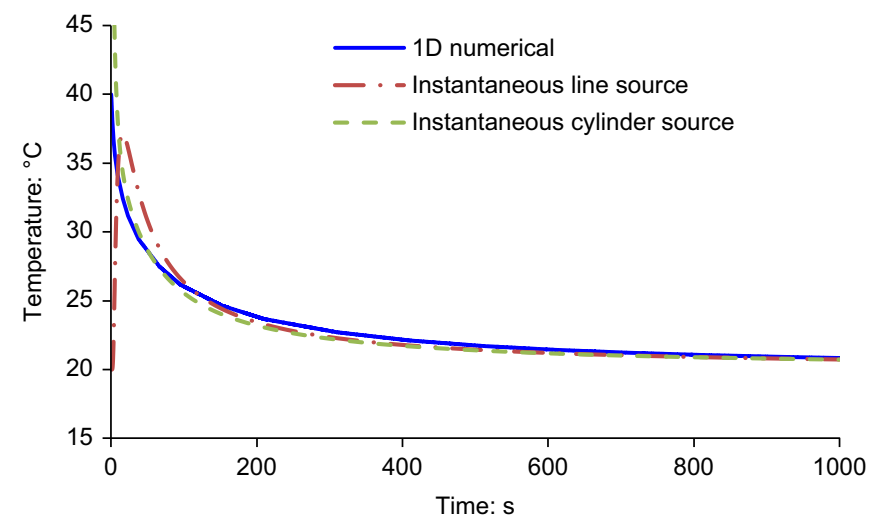

(a)

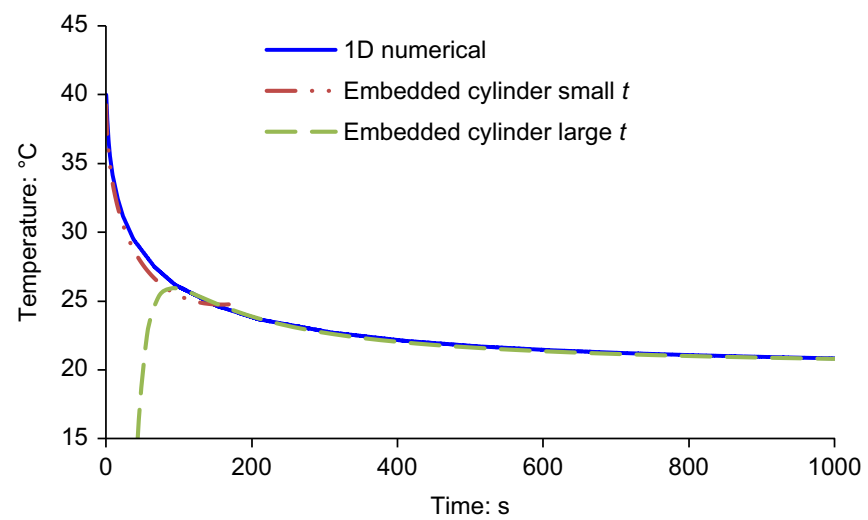

(c) (b) an instantaneous heat release along the surface of a cylinder inside an infinite medium

(c) a perfectly conducting cylinder inside an infinite medium with an initial temperature different to that of the surrounding medium.

\section{Instantaneous heat release along a line inside} an infinite medium

Instantaneous energy release considers a fixed amount of energy, in this case per length, released into the soil along a line. The analytical solution for this is (see section 10.3 of Carslaw \& Jaeger (1959))

$$
T(r, t)=T_{0}+\frac{H_{0} / L}{4 \pi k t} \exp \left(-\frac{c_{\mathrm{p}} \rho r^{2}}{4 k t}\right)
$$

where $T_{0}$ is the initial temperature and $H_{0} / L$ is the heat release per length. Given that the T-CPT cone is made of steel and is therefore significantly more conductive than the medium, the temperature where $r=r_{\mathrm{CPT}}$ (where $r_{\mathrm{CPT}}$ is the radius of the T-CPT cone) is representative of the temperature within the T-CPT cone. This method is presented in Figs 16(a) and 16(b), shown alongside numerical results, for the conditions given in the section entitled 'Interpretation model verification'.

By first moving the initial temperature to the left-hand side and taking the natural logarithm of the equation, this yields

$$
\ln \left(T-T_{0}\right)=\ln \left(\frac{H_{0} / L}{4 \pi k}\right)-\ln (t)-\frac{c_{\mathrm{p}} \rho r^{2}}{4 k t}
$$

The last term is only significant at small timescales (e.g. $<100 \mathrm{~s}$ for typical soils) and large radii. At small radii - for example the outer surface of a T-CPT cone, the thermal conductivity can be determined from data with a large enough timescale, that has a linear part of a $\ln \left(T-T_{0}\right)-\ln (t)$ plot, where the gradient should be

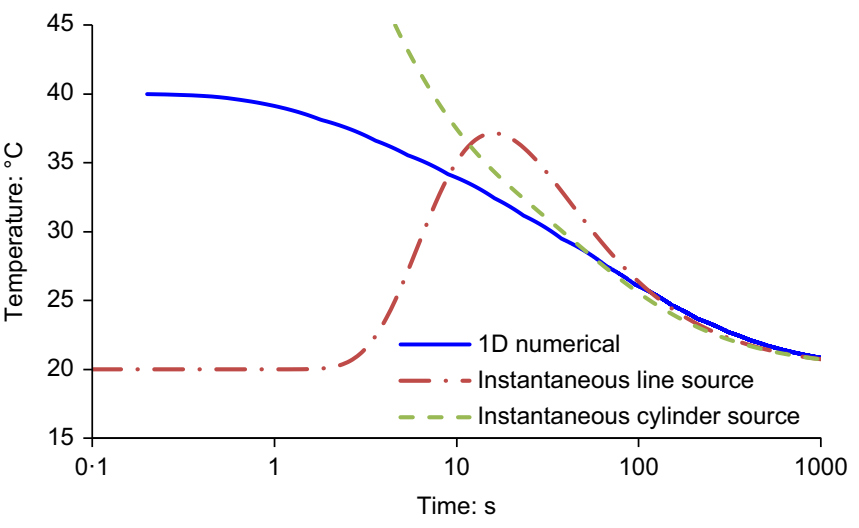

(b)

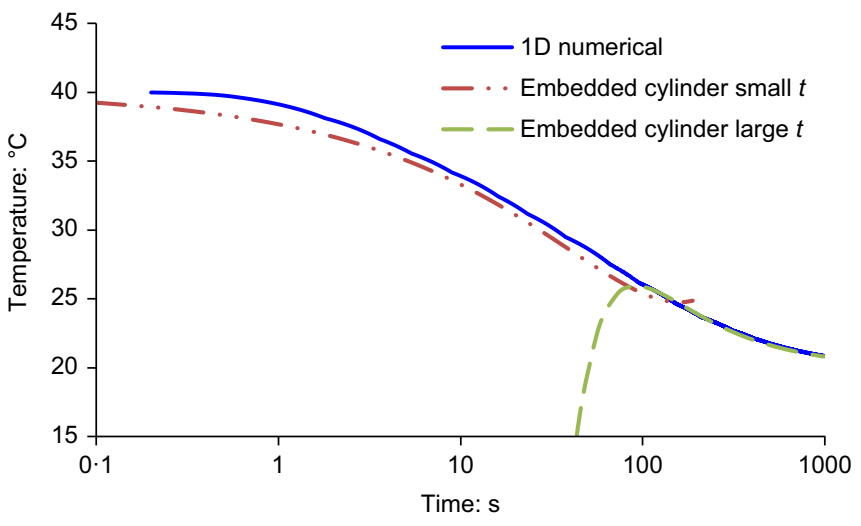

(d)

Fig. 16. The 1D axisymmetric numerical and analytical temperature of the T-CPT cone against time: (a) the two instantaneous heat source solutions against time; (b) the two instantaneous heat source solutions against time in log coordinates; (c) the embedded perfectly conductive cylinder solutions against time; and (d) the embedded perfectly conductive cylinder solutions against time in log coordinates 
exactly -1 , or directly from the following equation

$$
k=\frac{H_{0} / L}{4 \pi \exp \left[\ln \left(T-T_{0}\right)+\ln (t)\right]}=\frac{H_{0} / L}{4 \pi\left[t\left(T-T_{0}\right)\right]}
$$

This requires the accurate determination of $T_{0}$, which can be determined by any one of three methods: (a) waiting until full thermal dissipation; $(b)$ waiting until most of the temperature has dissipated and plotting $T-\sqrt{t}$ where the extrapolated $y$-intercept gives a good estimate of $T_{0}$; or $(c)$ by exploiting the gradient equalling -1 , giving

$$
T_{0}=\frac{t_{1} T_{1}-t_{2} T_{2}}{t_{1}-t_{2}}
$$

where the subscript 1 relates to the earlier time and 2 the later, and waiting until the value converges, which coincides in time with the linear portion of the $\ln \left(T-T_{0}\right)-\ln (t)$ plot.

The amount of energy instantly released is defined by the heat content in the T-CPT cone

$$
H_{0} / L=\left(T_{\max }-T_{0}\right) c_{\mathrm{p} \text {,steel }} \rho_{\text {steel }} A_{\text {steel }}
$$

where $A_{\text {steel }}$ is the cross-sectional area of steel in the T-CPT cone and $T_{\text {max }}$ is the maximum recorded temperature.

\section{Instantaneous heat release along the surface of a cylinder inside an infinite medium}

An instantaneous energy release considers a fixed amount of energy, again per length, released into the soil along the surface of a cylinder. The analytical solution for this is (see section 10.3 of Carslaw \& Jaeger (1959))

$$
T(r, t)=T_{0}+\frac{H_{0} / L}{4 \pi k t} \exp \left[-\frac{c_{\mathrm{p}} \rho\left(r^{2}+r_{\mathrm{c}}^{2}\right)}{4 k t}\right] I_{0}\left(-\frac{c_{\mathrm{p}} \rho r r_{\mathrm{c}}}{2 k t}\right)
$$

where $r_{\mathrm{c}}$ is the radius of the cylinder and $I_{0}$ is the modified Bessel function of the first type and order 0 . This method is also shown in Figs 16(a) and 16(b).

Again, by moving the initial temperature to the left-hand side and taking the natural logarithm of the equation and recognising that all terms but the first are insignificant at large times yields equation (8).

\section{Perfectly conducting cylinder inside an infinite medium}

This situation considers a cylinder of material inside an infinite medium (of another material). The cylinder is assumed to be perfectly conductive (reasonable when the material of the cylinder has a significantly higher thermal conductivity than that of the infinite medium), and is assumed to have a different initial temperature. It is also assumed that there is a perfect thermal contact between the materials.

The solution for the temperature of the cylinder is by way of a Laplace transform method and the inversion theorem (Jaeger, 1956)

$$
\begin{aligned}
& T(r, t)=T_{0}+\frac{4 \alpha_{l}\left(T_{\max }-T_{0}\right)}{\pi^{2}} \int_{0}^{\infty} \frac{\exp \left(-\tau u^{2}\right)}{u \Delta(u)} \mathrm{d} u \\
& \text { where } \Delta(u)=\left[u J_{0}(u)-\alpha_{l} J_{1}(u)\right]^{2}+\left[u Y_{0}(u)-\alpha_{l} Y_{1}(u)\right]^{2}
\end{aligned}
$$

where $J_{n}$ and $Y_{n}$ are Bessel functions of the first and second type of order $n$ and $u$ is the Laplace operator.

Two dimensionless numbers are used

$$
\begin{gathered}
\tau=\frac{\alpha t}{r_{\mathrm{c}}^{2}}=\frac{k t}{c_{\mathrm{p}} \rho r_{\mathrm{c}}^{2}} \\
\alpha_{l}=\frac{2 \pi c_{\mathrm{p}} \rho r_{\mathrm{c}}^{2}}{S}
\end{gathered}
$$

where $\alpha$ is the thermal diffusivity $\left(k / c_{\mathrm{p}} \rho\right)$ and $S$ is the heat content per unit length - that is, for the T-CPT cone, $S=c_{\mathrm{p} \text {,steel }} \rho_{\text {steel }} A_{\text {steel }}$.

The solution is not possible to evaluate analytically, but there are simpler expressions which are valid at small and large values of $\tau$, and therefore $t$ (Jaeger, 1956). These solutions are based upon expansions of series and therefore have a defined level or error. For small values of $t$

$$
T=T_{0}+\left(T_{\max }-T_{0}\right)\left[1-\frac{2 \alpha_{l}}{\pi^{1 / 2}} \tau^{1 / 2}+\alpha_{l}\left(\alpha_{l}-\frac{1}{2}\right) \tau+O\left(\tau^{3 / 2}\right)\right]
$$

where the $O($ ) function is used to indicate the level of error in the curtailed series. For larger values of $t$

$$
\begin{aligned}
T= & T_{0}+\left(T_{\max }-T_{0}\right)\left[\frac{1}{2 \alpha_{l} \tau}-\frac{\alpha_{l}}{4 \alpha_{l}^{2} \tau^{2}}-\frac{\left(\alpha_{l}-2\right)}{4 \alpha_{l}^{2} \tau^{2}}\right. \\
& \left.\times\left(\ln \frac{4 \tau}{2 \cdot 711}-1\right)+O\left(\tau^{-3} \ln \tau\right)\right]
\end{aligned}
$$

To show the connection to the previous approximate solutions (e.g. equations (8) and (11)), $S$ is found in the denominator of $\alpha_{l}$ and $H_{0} / L=S\left(T_{\max }-T_{0}\right)$.

Both of these solutions are presented in Figs 16(c) and 16(d), shown alongside the other methods and the numerical results.

Starting then with equation (16) and recognising that at very large values of $\tau$ the first two terms dominate, yields the same result as equation (8).

Taking equation (15) and recognising that at very small values of $t$ only the first two terms are significant, yields after substitution from the dimensionless numbers (equations (13) and (14)) and rearrangement into normalised temperature

$$
T_{\text {norm }}=\frac{T_{\max }-T}{T_{\max }-T_{0}}=1-\frac{T-T_{0}}{T_{\max }-T_{0}}=\frac{4 r_{\mathrm{c}}\left(\pi k \rho c_{\mathrm{p}}\right)^{1 / 2}}{S} t^{1 / 2}
$$

Taking the differential of the normalised temperature against the square root of time and rearranging, yields the volumetric heat capacity

$$
C=\rho c_{\mathrm{p}}=\left(\left.\frac{\mathrm{d} T_{\text {norm }}}{\mathrm{d} \sqrt{t}}\right|_{t \rightarrow 0} \frac{S}{4 r_{\mathrm{c}}}\right)^{2} \frac{1}{\pi k}
$$

This indicates that volumetric heat capacity can be determined by taking the tangent (gradient) of a $T_{\text {norm }}-\sqrt{t}$ plot from the origin.

\section{Interpretation model}

Adding T-CPT cone-specific calibration factors to both equations (8) and (18) allows non-perfect cylinders (i.e. with internal voids), non-perfectly conducting cylinders or non-infinite line sources to be used. The interpretation model resulting is given in the section entitled 'Proposed interpretation model'.

\section{NOTATION}

$A_{\text {steel }}$ cross-sectional area of steel in cone penetrometer

$C$ volumetric heat capacity

$c_{\mathrm{p}}$ specific heat capacity

$f_{\mathrm{HC}}$ calibration factor for heat capacity

$f_{\text {TC }}$ calibration factor for thermal conductivity

$\operatorname{grad}_{T} \operatorname{gradient}$ at the origin from a $T_{\text {norm }}-\sqrt{t}$ plot

$H_{0} / L$ heat release per length

$I_{0}$ modified Bessel function of the first type and order 0

$i_{T} \quad y$-intercept from $\ln \left(T-T_{0}\right)-\ln (t)$ plot

$J_{n}$ Bessel function of the first type of order $n$

$k$ thermal conductivity

$n$ counter for data reading

$O($ ) level of error

$Q$ heat flux per metre

$r$ radial coordinate

$r_{\mathrm{c}}$ radius of cylinder

$r_{\mathrm{CPT}}$ radius of cone penetrometer

$S$ heat content per unit length

$T$ temperature

$T_{0}$ initial in situ temperature

$T_{\max }$ maximum recorded temperature

$T_{\text {norm }}$ normalised temperature 
$t$ time

$u$ Laplace operator

$Y_{n}$ Bessel function of the second type of order $n$

$\alpha$ thermal diffusivity $\left(k / c_{\mathrm{p}} \rho\right)$

$\alpha_{l}$ dimensionless number defined in equation (14)

$\rho$ density

$\tau$ dimensionless number defined in equation (13)

\section{REFERENCES}

Akrouch, G. A., Briaud, J. L., Sanchez, M. \& Yilmaz, R. (2016). Thermal cone test to determine soil thermal properties. J. Geotech. Geoenviron. Engng 142, No. 3, 04015085.

ASTM (2014). D5334 - 14: Standard test method for determination of thermal conductivity of soil and soft rock by thermal needle probe procedure. West Conshohocken, PA, USA: ASTM International.

Carslaw, H. S. \& Jaeger, J. C. (1959). Conduction of heat in solids, 2nd edn. London, UK: Oxford University Press.

de Gijt, J. G. \& van Roekel, G. (1987). Cone penetration testing in relation to environmental problems. In Environmental technology: Proceedings of the 2nd European conference on environmental technology (eds K. J. A. de Waal and W. J. van den Brink), pp. 577-586. Dordrecht, the Netherlands: Martinus Nijhoff.

Ewen, J. \& Thomas, H. R. (1992). The thermal probe measurement of the thermal conductivity and drying rate of soil in the field. Geotech. Testing J. 15, No. 3, 256-263.
Farouki, O. T. (1981). Thermal properties of soils. Cold Regions Science and Engineering Laboratory Monograph 81-1. Hanover, NH, USA: US Army Corps of Engineers.

ISO (International Organization for Standardization) (2012). EN ISO 22476-1:2012 Geotechnical investigation and testing. Field testing. Part 1: Electrical cone and piezocone penetration test. Geneva, Switzerland: ISO.

Jaeger, J. C. (1956). Conduction of heat in an infinite region bounded internally by a circular cylinder of a perfect conductor. Aust. J. Phys. 9, No. 2, 167-179.

Mayne, P. W., Peuchen, J. \& Bouwmeester, D. (2010). Soil unit weight estimation from CPTs. In Proceedings of the 2nd international symposium on cone penetration testing, CPT'10 (eds P. K. Robertson and P. W. Mayne), vol. 2, pp. 169-176. Huntington Beach, CA, USA: CPT'10 Organizing Committee.

Post, M. L. \& Nebbeling, H. (1995). Uncertainties in cone penetration testing. In Proceedings of the international symposium on cone penetration testing, CPT'95, vol. 2, pp. 73-78. Linkoping, Sweden: Swedish Geotechnical Society.

Vardon, P. J., Baltoukas, D. \& Peuchen, J. (2018). Example calculation sheet for thermal cone penetration test (T-CPT). Delft, the Netherlands: Delft University of Technology. See https:// pure.tudelft.nl/portal/en/publications/example-calculation-sheetfor-thermal-cone-penetration-test-tcpt(4244ff3c-df3e-4946-a0cb6a65face2947).html, https://doi.org/10.4121/uuid:c2e3cee30b22-474a-9d4e-7712335a0d00 (accessed 28/08/2018). 